authors conclude that this protein might prove to be a useful noninvasive biomarker for prostate cancer recurrence.

Original article Banyard J et al. (2007) Collagen XXIII expression is associated with prostate cancer recurrence and distant metastases. Clin Cancer Res 13: 2634-2642

\section{JM-27 levels can distinguish between different forms of benign prostatic hyperplasia}

Although benign prostatic hyperplasia $(\mathrm{BPH})$ has sometimes been considered to be a single disease with varying symptoms, recent studies have shown differences at the gene expression level between individuals with moderate to severe $\mathrm{BPH}$ symptoms and those with histological disease. JM27 is an androgen-regulated gene that is highly expressed in patients with symptomatic $\mathrm{BPH}$. A study conducted by Cannon et al. evaluated the role of JM27 in distinguishing patients with different forms of $\mathrm{BPH}$.

In order to measure the presence of the JM27 protein in the serum of 85 patients, a JM-27 enzyme-linked immunosorbent assay was developed using an anti-JM-27 monoclonal antibody. The patients were divided into three groups: patients with asymptomatic BPH (group A, $n=29$ ); patients with symptomatic BPH (group B, $n=39$ ); and patients with prostate cancer (group C, $n=17$ ). The difference in serum JM-27 levels between patients from group $B$ and patients from groups $A$ and $C$ was highly significant $(P<0.0001)$. Estimates of the sensitivity and specificity of the assay for distinguishing symptomatic from asymptomatic patients were $90 \%$ and $77 \%$, respectively. Prostate volume did not affect the outcome of the assay.

Although further research is required, this study suggests that JM-27 could represent the first serum-based marker for symptomatic BPH.

Original article Cannon GW et al. (2007) A preliminary study of JM-27: a serum marker that can specifically identify men with symptomatic benign prostatic hyperplasia. J Urol 177: 610-614

\section{Low testosterone levels in boys conceived using intra-cytoplasmic sperm injection}

Boys conceived using intra-cytoplasmic sperm injection (ICSI) have lower serum testosterone levels than their naturally conceived counterparts, according to a recent study from Denmark where $1.5 \%$ of all children are conceived using ICSI.

ICSI is an increasingly popular technology for overcoming male infertility. Previous evidence has suggested that impaired testicular function might be passed on from father to son when this technique is used, but the possibility of impaired Leydig cell function in ICSI offspring has not previously been investigated.

Mau Kai and colleagues compared serum levels of luteinizing hormone, follicle stimulating hormone, sex-hormone-binding globulin, inhibin $\mathrm{B}$, and testosterone in boys conceived using ICSI $(n=125)$, boys conceived using in vitro fertilization $(n=124)$, and boys conceived naturally $(n=933)$ in a prospective, longitudinal cohort study. Measurements were taken at birth and, in most cases, at 3 months of age.

The results showed that boys conceived using ICSI had significantly lower serum testosterone levels compared with boys conceived naturally $(P<0.001)$, and a higher luteinizing hormone to testosterone ratio $(P=0.001)$. By contrast, both serum testosterone levels and luteinizing hormone to testosterone ratio were normal in the boys conceived using in vitro fertilization because of female infertility factors.

These results suggest that boys conceived using ICSI might have a subtle impairment of Leydig cell function that could have been inherited from their fathers. The authors conclude that, given the increasing popularity of ICSI, these findings should raise concern, although their clinical relevance remains unclear.

Original article Mau Kai C et al. (2007) Reduced serum testosterone levels in infant boys conceived by Intracytoplasmic sperm injection (ICSI). J Clin Endocrinol Metab 92: 2598-2603 